\title{
Asset Distribution and Peerless Selection Approach for LTE based Multifarious VANET Networks
}

\author{
Arjun Arora*, Nitin Rakesh \\ Department of Computer Science and Engineering \\ Amity School of Engineering and Technology, Amity University, Noida, Uttar Pradesh, India \\ E-mail: nrakesh@amity.edu \\ "Corresponding author: a.arora@ ddn.upes.ac.in \\ K. K. Mishra \\ Department of Computer Science \\ Motilal Nehru National Institute of Technology, Allahahbad, Uttar Pradesh, India \\ E-mail: kkm@mnnit.ac.in
}

(Received July 26, 2018; Accepted October 18, 2018)

\begin{abstract}
Vehicular ad-hoc networks (VANET) over the past decade have been always part of research and enthusiasts, bringing in a lot of attention towards them. But with the tremendous swell in the imposition for mobile data VANETs are struggling to meet up. Vehicle to Vehicle (V2V) type of transmission is considered a very successful methodology for assuming trustworthy communication amongst vehicles participating in such communications. In the case of multifarious networks also considering the typical cellular network in which cellular links co-exist with V2V communication by making use of the same resources available in a given spectrum, resulting in a complex scenario. Therefore, it is a challenging issue to tackle with asset distribution and peerless selection. In this paper, a scheme is proposed which shows that peerless selection approach and asset distribution can be used as mixed user utility maximization with consideration of a joint network with the delay in transmission and reduction in power. To minimize the complicacy of computation a distributed algorithm is proposed which boils down towards a near-perfect solution by making use of the Lagrangian technique. The numerical analysis shows the extraordinary gains in throughput can be achieved specially with larger networks. The throughput of the network is also improved because of reduction in power.
\end{abstract}

Keywords- VANET, Networking, LTE.

\section{Introduction}

Over a few years, there has been observed a humungous increase in demand for mobile traffic as well as high speed access for data. As per the graphical network index is given by CISCO Systems (Al-Kubati et al., 2015), the total number of locomotive traffic globally is bound to grow ten times by 2019 , approximately going upto 25 exa-bytes every month. Also, smart communication devices in totality are responsible for the generation of $97 \%$ of traffic data globally. Such huge amounts of demand for data lays a very substantial impact on conventional communication over cellular network owing to the limited resource spectrum specially for users at the edge of a cell or areas of the network where there is fading in such cases users are hardly able to enjoy connectivity. This makes it extremely important to find a solution to the problem of limited spectrum resource and need of a new architecture as well as selection of appropriate approach. With the advent of intelligent computing VANETs have attracted a lot of attention from researchers as well as government organizations (Al-Kubati et al., 2014; Alshaer, 2015). VANETs can be formed by making vehicles to communicate with vehicles (V2V) or making vehicles communicate with infrastructure (V2I) delivering information about vehicle status, 
International Journal of Mathematical, Engineering and Management Sciences

Vol. 4, No. 1, 27-40, 2019

https://dx.doi.org/10.33889/IJMEMS.2019.4.1-003

position, emergency signal etc. (Arora et al., 2018). Even though VANETs are so much in demand the need of user cannot be fulfilled because of throughput and delay. Communication among devices often termed as Device to Device (D2D) communication enables direct transmission amongst physically near-by users by making use of similar resources as used by regular users of a cellular network. This method is considered to be promising and reliable in providing connectivity (Balasubramanian et al., 2008; Bai et al., 2015; Bazzi et al., 2016). With the introduction of Device to Device communication there arises an event for V2V support with the strict quality of service standards. The working of D2D is replicated in V2V by making use of the same spectrum resources as in the case of cellular networks (Bazzi et al., 2016). The benefits of $\mathrm{V} 2 \mathrm{~V}$ style communication is not only limited to enhancing the structural capability but also it can help improve on energy efficiency, fairness and delay in a given network. Some prominent related research work has been done on LTE-VANET multifarious networks over the past decade. Researchers analysed the impact of floating car data in comparison to human -2-human traffic in case of a cellular VANET multifarious network and make the design of a simple floating car data scheme which is then evaluated with the input of human-2-human traffic. In (Bessani et al., 2013; Bitam and Mellouk, 2014) the influence of combining spectrum bandwidth is assessed in the case of multifarious networks. Researchers investigated varied graph colouring schemas for asset sharing in order to solve multiple joint blocks of resources (Elazab et al., 2017) and transmission of power allocation issues. Researchers have shown geographical based re-use of cellular user schemes which aims at increasing the total sum rate, at the same time decreasing the rate of achievement (Di Felice et al., 2012). In the paper of George and Venugopal (2012) a location based asset distribution scheme for D2D communication which can be implemented in V2V environment is developed. An algorithm for asset distribution is used in order to reduce cellular resource consumption (Han et al., 2010). In paper of Jemma et al. (2015) researchers have developed a joint asset distribution and power limit algorithm in order to increase the total rate of the sum for cellular users which generates latency as well as the reliability of vehicles. Khaitiyakun et al. (2014) developed a framework over mobile users association and mode of transmission which switches between direct and D2D for improvement of spectrum and energy efficiency. Malandrino et al. (2012) researchers have proposed election mechanism for the nomination of a cluster head which is used effectively manage all the sub-clusters formed within a given VANET. This ultimately guarantees communication of multimedia content over a partially distant group of vehicles. A cloud based distribution scheme is studied so that safety messages can be sent over the cloud server to the suitable mobile gateway and related messages be distributed within the vehicles in the vicinity in-case of V2V (Mershad and Artail, 2013). In paper (Mershad et al., 2012a) authors have debated on an algorithm on fuzzy logic, which has gateway draft from a given VANET to an advanced LTE infrastructure providing more safety and security in transportation, quality services and associated applications. Data offloading for traffic from LTE to VANET is assessed and formulated simply as maximum flow problem for optimization (Mershad et al., 2012b). In work of Mostafa et al. (2011), researchers have deployed an LTE-VANET multifarious network in order to achieve an efficient distribution of data, using which the game theory concept is combined and applied in order to provide the simulation of vehicles for joining condition and maximizing an efficient rate of data in transfer. With huge V2V communication links it results in a more complex interference scenario. The cellular user suffers interference generated from $\mathrm{V} 2 \mathrm{~V}$ communication and base stations $\left(B_{S}\right)$, severely disturbs the signals received and their quality owing to re-use of the spectrum. The scenario gets worse due to high fluctuation in V2V services causing in-efficient utilization of resources. The occurrence of idle $\mathrm{B}_{\mathrm{S}}$ and vehicular transmissions $\left(V_{T}\right)$ results in wastage of power with over interference ultimately resulting in deterioration of performance in a given network. Turning off idle $B_{S}$ and $V_{T}$ 
International Journal of Mathematical, Engineering and Management Sciences

Vol. 4, No. 1, 27-40, 2019

https://dx.doi.org/10.33889/IJMEMS.2019.4.1-003

benefits in saving power as well as helps in avoiding interference. Using this observation as the basis this paper makes an attempt to explore the perfect peerless selection scheme and asset distribution in order to provide improved capacity. The proposed schema can be deployed in various situations especially in case of emergency, providing high quality and application service for users of vehicles. The highlights of this paper are briefed in the following mentioned points:

a. This paper proposes a peerless selection approach and asset distribution scheme in LTEVANET multifarious network. In such a network vehicular users take decisions for selection of an appropriate scheme and capture corresponding resources between LTEcommunication and device to device based vehicle to vehicle communication. Cellular users in such a case need to choose their own peerless suited $B_{s}$. Maximization of the utility problem is made by combining all the users in joint consideration with topology, delay in transmission in $\mathrm{V} 2 \mathrm{~V}$ and minimization of power. This serves as a standard using which other scenarios can be assessed having a much more complicated network.

b. In order to avoid computation complexity of higher magnitude for large scale networks, an algorithm is devised which only takes two steps i.e. double decomposition is taken into consideration to find solution to maximum utility problem taking into account all $\mathrm{B}_{\mathrm{S}}$ and all the vehicle transmissions are made to work in active mode with all the idle $B_{S}$ and $V_{T}$ being switched off, this results in higher throughput performance because of interference minimization.

c. Numerous numerical situations are conducted in order to assess the proposed algorithm under varied conditions of traffic. Results of various simulations show that a huge change in throughput can be gained by making use of the algorithm proposed especially with the increase in the network. It may be noted that simulations also show that throughput can also be improved re-mark ably by exploiting reduction power.

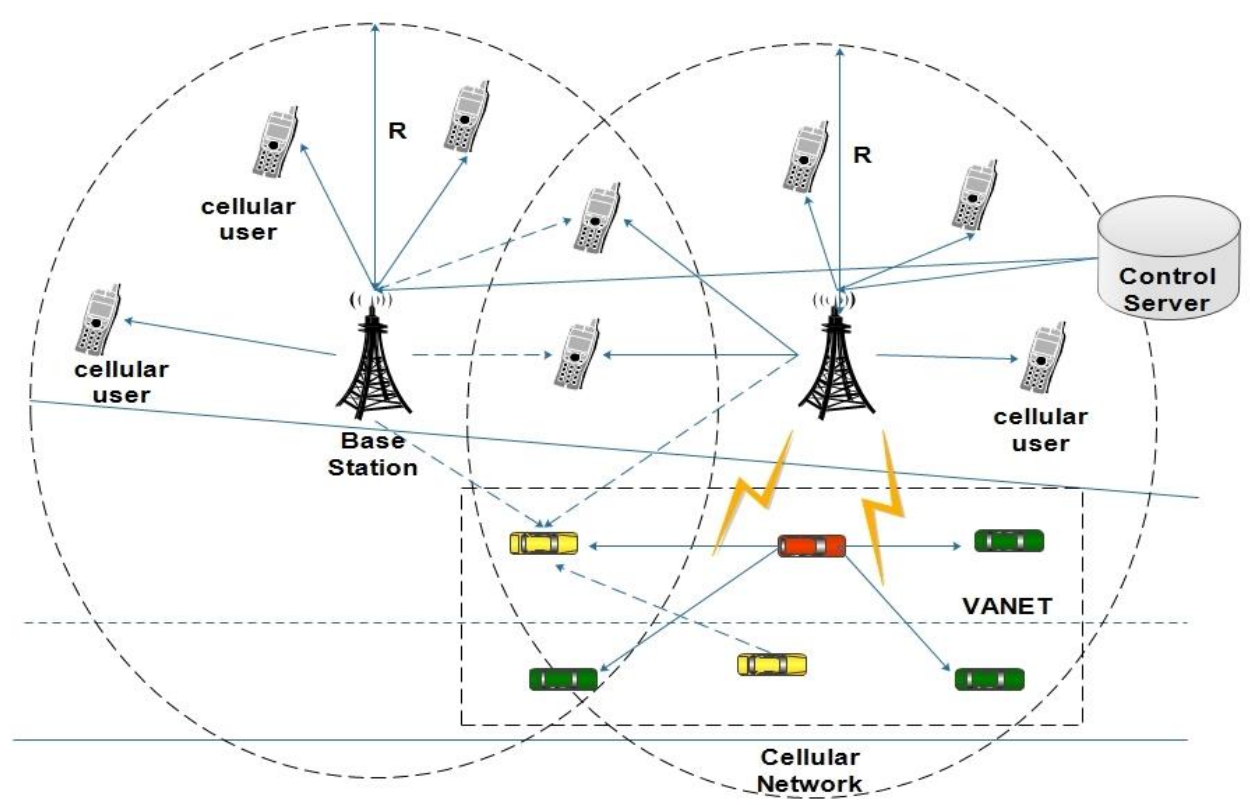

Figure 1. Graphical model for cellular- VANET with divergent network 
International Journal of Mathematical, Engineering and Management Sciences

Vol. 4, No. 1, 27-40, 2019

https://dx.doi.org/10.33889/IJMEMS.2019.4.1-003

\section{Network Model and Formulation of Problem}

In this section, the network model is discussed along-with network topology, interference, delay in transmission and problem formulation is made. This paper mainly targets on the down-link aspect. Now if we scrutinize an LTE-VANET multifarious network, which includes typical communication links as in a cellular network with a given collection of Device to Device (D2D) based V2V as shown in Figure 1. The $B_{S}$ simply gets hold of the central part of a given macro-cell having radius $(r)$ and cellular users are haphazardly scattered in the given cell. Through this cell a road crosses and all the vehicles which are receiving and are equipped with user equipment can communicate amongst the vehicles in the neighbourhood and at the same time contain cellular interface in order to deploy an explicit communication with the cellular network. A majority of vehicles having both the types i.e. transmitting ones as well as receiving ones move towards the cell-block which is completely covered and has density of ' $\rho$ ' vehicles/meter. Taking into consideration ' $\gamma T_{N V}$ ', as vehicles transmitting $\left(V_{T}\right)$ are uniformly scattered on the straight road which includes selected $V_{T}$ and candidate $V_{T}$ and where $\gamma$ is the ratio of number of $V_{T}$ and total numbethe $\mathrm{r}$ of vehicles $\left(T_{N V}\right)$ on the given road. When each of the vehicular receivers $\left(\mathrm{V}_{\mathrm{R}}\right)$ communicate with its own $V_{T}$ then unselected $V_{T}$ are considered as candidate $\mathrm{V}_{\mathrm{T}}$. Due to time dependent different conditions of traffic a given candidate $V_{T}$ will turn into serving $V_{T}$ simply in order to provide wireless communication to surrounding $V_{R}$. There are two types of links which co-exist in case of cellular communication one between $B_{S}$ and cellular user and the other between D2D based V2V links amongst vehicles. The $V_{T}$ and its related $V_{R}$ form VANET which repeatedly use the same spectrum of cellular network. the Cellular users have access to only cellular network however $V_{R}$ have capability to switch from cellular to VANET and vice-versa. A single $V_{T}$ can distribute localised information with surrounding $V_{R}$ or send messages to the closest $B_{S}$ making use of air medium. Messages that are sent to the ground server, which collects and helps in keeping a record of current information regarding each and every $B_{S}$ and $V_{T}$ which are used in controlling D2D based V2V, $B_{S}$ operational switching and regulation of traffic. Consider that there are ' $\ell$ ' number of $B_{S}$ and $V_{T}$ given by $i=1, \ldots \ldots, \ell$. Cellular users and $V_{R}$ given by ' $m$ ' denoted as $j=1, \ldots \ldots, m$. Omnidirectional antennas transmitting with absolute power causing interference which is witnessed by a single cellular user with approximate measure from $\mathrm{B}_{\mathrm{S}}$ and $V_{T}$ because of spectrum re-use. In order to understand operational state of $B_{S}$ and $V_{T}$ we define an indicator ' $\beta_{k}{ }^{\prime}$ as:

$\beta_{k}= \begin{cases}1, & B_{S} \text { or } V_{T} \\ 0, & B_{S} \text { or } V_{T} \text { is in active state }\end{cases}$

Throughput for one cellular user directly depends on the function of signal interference and noise ratio (SINR) in terms of bandwidth allocation and spectrum efficiency, which is in line with the formula given by Shannon. SINR is shown by $R_{i j}$ and is computed as:

$R_{i j}=\frac{\beta_{k} T_{P_{i}} C_{A_{i j}}}{\sum_{M=1}^{M} \beta_{M} P_{M} H_{M j}+\alpha^{2}}$

In the above equation (2) $T_{P_{i}}$ is transmitted power, $C_{A_{i j}}$ is channel attenuation, assuming the channel characteristic to slowly fade and then association process is executed over a short period of time. $\sum_{M=1, M \neq i}^{m} \beta_{M} P_{M} H_{M_{j}}$ is the received interference in totality from $B_{S}$ and $V_{T}$ and $\alpha^{2}$ is simply the noise power (thermal). All cellular users and vehicles typically in an overlapped region selection approach for access is conducted with the ultimate aim being to bring out the 
International Journal of Mathematical, Engineering and Management Sciences

Vol. 4, No. 1, 27-40, 2019

https://dx.doi.org/10.33889/IJMEMS.2019.4.1-003

peerless selection approach and asset distribution, when selected amount of $B_{S}$ or $V_{T}$ are switched off in order to maximise collective user utility. As we are aware that in VANETs there is high movement of vehicles due to which the delay impact between two vehicles in concern should guarantee V2V services and applications. Delay in transmission can be defined as passing time for a given message from source to destination for a given VANET, this is closely related to distance of transmission amongst two vehicles. Delay in performance is meagrely satisfied when distance between source and destination is very long. Thus, it becomes crucial to disrupt the relation between transmission delay and distance respectively. By making uses of theoretical equations (3) and (4) a relationship can be declined between transmission delay and distance. Total time available is divided into time slots $(t \in n)$, transmission delay is computed only along one direction. For each given time slot vehicles are located using Poisson Point. ' $Y_{D}$ ' denotes vehicle location $\mathrm{D}$ of a vehicle. Transmission distance between two vehicles $\mathrm{A}$ and $\mathrm{B}$ is given by $L_{A, B}=\left\|Y_{A}-Y_{B}\right\|$, here $\|$.$\| is Euclidean norm. Then delay in transmission from a vehicle A to$ vehicle $B$ is

$D_{T}(A, B)=T(A)-T(B)=\sum_{g=A}^{B-1}[T(g+1)-T(g)]$

Now here $D_{T}(A, B)$ is delay in transmission from vehicle $\mathrm{A}$ to $\mathrm{B}$. $\mathrm{T}(\mathrm{g})$ denotes time at which a vehicle $\mathrm{g}$ receives message. A given vehicle $\mathrm{g}$ is located within interval position $Y_{A}$ to $Y_{B}$. Now assuming $C_{1}(t)$ and $C_{0}(t)$ denote probability of connection and disconnection at given instance of time $(\mathrm{t})$ and we know that vehicles are using poisson point for marking their location which is static at any point in time, $C_{1}(t)$ and $C_{0}(t)$ are absolute values of time $(t)$ therefore can be written as $C_{1}$ and $C_{0}$. The relationship between delay and distance with respect to transmission can be stated as a linear relationship, $Q=D_{T}(A, B) / L_{A, B}$ where $\mathrm{Q}$ is constant. Now in order to prove the above mentioned statement ergodic theorem proposed in (Nam et al., 2015) is used. $\left\{Z_{m, n}\right\}$ is suppose a collection of random variables, satisfying $0 \leq m \leq n$. Then (Nam et al., 2015) makes assumptions that

(i) $Z_{l, n} \leq Z_{l, n}+Z_{m, n}$ when $0 \leq l \leq m \leq n$.

(ii) $\left\{Z_{m+1, n+1}, 0 \leq m<n\right\}$ is same as $\left\{Z_{m, n}, 0 \leq m<n\right\}$.

(iii) For every $\mathrm{n}, F\left|Z_{0, n}\right|<\infty$ and $F\left(Z_{0, n}\right) \geq-h n$ where $h$ is constant. All the above conditions when satisfy it concludes that $Z=\lim _{n \rightarrow \infty} Z_{0, n} / n$ is true.

$$
\begin{aligned}
& F\left(D_{T}(g, g+1)\right)=\int_{0}^{\infty} P\left\{D_{T}(g, g+1)=t\right\} d t=\int_{0}^{\infty}\left(C_{o}\right)^{t-1} C_{1} d t=-\frac{C_{1}}{C_{0} \ln \left(C_{0}\right)} \\
& Q=\lim _{L_{A, B} \rightarrow \infty} \frac{F\left(D_{T}(A, B)\right)}{L_{A, B}}=\lim _{L_{A, B \rightarrow \infty}} \frac{F\left(\eta_{A, B}-1\right) F\left(D_{T}(g, g+1)\right)}{L_{A, B}}=-\frac{\lambda C_{1}}{C_{0} \ln \left(C_{o}\right)}
\end{aligned}
$$

Now in order to prove that the model proposed in this paper satisfies the theorem mentioned in (Nam et al., 2015), we assume that all vehicles present in 1-dimensional space inside a triangle constituting of $1, m$ and $n$. messages delivered from $\omega_{l}$ to $\omega_{n}$ and then from $\omega_{m}$ to $\omega_{n}$ which means $D_{T_{l, n}} \leq D_{T_{l, m}}+D_{T_{m, n}}$. If vehicles $1, m$ and $n$ lie on a straight line then $D_{T_{l, n}}=D_{T_{l, m}}+$ $D_{T_{m, n}}$ this satisfies the condition one. Second condition i.e. vehicle dissemination is based on poisson point which is static having density $\lambda$ at time $(t)$ is automatically satisfied. Last condition $F\left|D_{T}(0, k)\right|=F\left(n_{0, k}\right) F\left(D_{T}(g, g+1)\right)$ where $n_{0, k}$ is number of vehicles between internal $V_{0} t o V_{n}$. Now as vehicles have Poisson distribution density $\lambda, F\left(n_{0, k}\right)=L_{0, k} \cdot \lambda-1$. Then 
International Journal of Mathematical, Engineering and Management Sciences

Vol. 4, No. 1, 27-40, 2019

https://dx.doi.org/10.33889/IJMEMS.2019.4.1-003

$F\left(D_{T}(g, g+1)\right)$ needs to prove that $P\left\{D_{T}(g, g+1)<\infty\right\}=1$. Now the above mentioned statement can be re-written as $1-P\left\{D_{T}(g, g+1)\right\}=\infty$ and this can be re-written as $1-C_{0}^{\infty}=$ $>1$ this concludes that $F\left\{D_{T}(g, g+1)<\infty\right\}$. When $S>0, F\left(D_{T}(0, n)\right)$ is positive (+) more than any negative (-). This satisfies the third condition as well. From the above made statements we can obtain the conclusion that $Q=\lim _{L_{A, B} \rightarrow \infty} D_{T}(A, B) / L_{A, B}$. The expected value of $\mathrm{D}(\mathrm{g}, \mathrm{g}+1)$ is calculated by equation (4) and completed expression for $\mathrm{Q}$ is given by equation (5). Vehicles are assigned using poisson point with density $\lambda$ then $L_{A, B}$ is distributed exponentially with density $\lambda$.

$C_{1}=P\left(L_{A, B} \leq r\right)=1-e^{-\lambda r}$

where $r$ is transmitter range

$C_{0}=1-C_{1}=e^{-\lambda r}$

The relationship between transmission distance and delay in transmission is given as:

$D_{T}(A, B)=-\frac{\lambda C_{1}}{C_{0} \ln \left(C_{o}\right)} L_{A, B}=\frac{1-e^{-\lambda r}}{r e^{-\lambda r}} L_{A, B}$

Now assuming a single hop transmission delay for Device to Device (D2D) based V2V is given by $D_{T}$ then the distance of transmission between $V_{T}$ and $V_{R}$ is less than $L_{T}$ and is given by:

$L_{t}=\min \left\{\frac{r e^{-\lambda r}}{1-e^{-\lambda r}} D_{t, r}\right\}$

Any vehicle in the network can opt Device to Device (D2D) based V2V communication however only vehicles which fulfil the criteria that distance from $V_{T}$ is within $L_{T}$ can make the selection of their own mode of transmission between VANET and cellular network. The throughput that can be achieved by one cellular user is:

$r_{i j}=S_{i j} \log \left(1+U_{i j}\right)$

Here, $S_{i j}$ is bandwidth for one cellular user. Only one $B_{S}$ or $V_{T}$ is selected after processing of access request. Association indicator is proposed to show the relationship as:

$\eta_{i j}=\left\{\begin{array}{rr}1, & B_{S} \text { selected by } V_{R} \\ 0, & \text { otherwise }\end{array}\right.$

The main aim is to maximise the collective network benefits which include cellular users and $V_{R}$. If collective throughput is taken as objective users having exceptional channel aspect will gain most bandwidth while faded users may not even get satisfactory service, which would lead to unbalanced allocation of resources. Exceeding to the linear function log function is regarded as an appropriate fitness function, which in true terms would imitate back the required level of satisfaction owing to its continuity. This property indicates just bandwidth distribution among cellular users and $V_{R}$. This results in user utility maximization problem to be formed as: 
International Journal of Mathematical, Engineering and Management Sciences

Vol. 4, No. 1, 27-40, 2019

https://dx.doi.org/10.33889/IJMEMS.2019.4.1-003

$$
\begin{aligned}
& \max _{S_{i j} \alpha_{i} \eta_{i j}} \sum_{j=1}^{N} \log \left(\sum_{i=1}^{M} \eta_{i j} \alpha_{i} S_{i j} \log \left(1+U_{i j}\right)\right. \\
& \sum_{j=1}^{N} \eta_{i j} S_{i j} \leq G_{i} \\
& \sum_{j=1}^{N} \eta_{i j} r_{i j} \leq H_{i} \\
& \sum_{j=1}^{N} \eta_{i j}=1, \forall j \in \text { cellular user } \\
& \sum_{i=1}^{M} \eta_{i j}=1, \forall j \in V_{R} \\
& \left\|\omega_{i}-\omega_{j}\right\| \leq L_{t}, \quad \forall j \in V_{T} \\
& \alpha_{i} \in\{0,1\}, \quad \forall i \\
& \eta_{i j} \in\{0,1\}, \quad \forall i \forall j
\end{aligned}
$$

Equation (13) stresses the fact that the total bandwidth utilization for a given $B_{S}$ should not be more than the bandwidth available $\left(G_{i}\right)$. Equation (14) explains that total capacity for a given $B_{S}$ achievable cannot exceed at any point in time with capacity of tolerance $\left(H_{i}\right)$, the rate being $r_{i j}$ is computed in equation (10). Equation (15) shows association of a single cellular user selection with only one $B_{S}$ at given instance of time. $M_{B_{S}}$ is simply the total number of $B_{S}$. Equation (16) define that $V_{R}$ serviced by corresponding $V_{T}$ should be located in the region having radius $L_{t}$ and $V_{T}$ is set of $V_{R}$. Equation (18) and (19) by way of indicators $\alpha_{i}$ and $\eta_{i j}$ show binary values. Now as $\alpha_{i}$ and $\eta_{i j}$ have binary values with $S_{i j}$ being a continuous variable, peerless selection is considered as a varied integer programming problem of non-linear type which is NP-hard (Puspitorini et al., 2017). $\alpha_{i}, \eta_{i j}$ and $S_{i j}$ are combined as constraints for resource and capacity making the entire scenario more complex. In order to solve such NP-hard problems brute-force cannot be used because complexity is very high. This makes the computation almost impossible even for a moderately sized network. Owing to the highly complex nature, it makes us to look for a dissemination algorithm, which has low complexity especially in case of larger networks.

\section{Asset Distribution and Peerless Selection Approach}

In order to minimize complexity in computing a two-step algorithm is proposed which formulates peerless selection approach and asset distribution as an aggregate of user's utility maximization problem taking into consideration that all the cellular $B_{S}$ and $V_{T}$ are in active state initially and reduction in power is conducted later on. Using Kuhn-Tucker the optimization problem cannot be resolved and also because it is not convex in nature as it has binary values. However, convex optimization does provide a durable method for approximation by means of dual decomposition. In comparison to giving a common solution having huge amounts of complexity, by making use of dual decomposition one can obtain a two-step solution with iteration in order to simplify the process. In the initial step we ignore the binary variable constraint. The bandwidth is allocated to all $B_{S}$ and $V_{T}$ after which from the pool of candidates only the one which has the highest rate is selected. In order to keep the errors minimum simple truncation is used in case of networking (Ramakrishnan et al., 2012). Lagrangian equation (20) for the main problem is given as: 
International Journal of Mathematical, Engineering and Management Sciences

Vol. 4, No. 1, 27-40, 2019

https://dx.doi.org/10.33889/IJMEMS.2019.4.1-003

$P=\left(S_{i j}, \lambda_{i}^{b}, \lambda_{i}^{r}\right)=-\sum_{i=1}^{M} \sum_{j=1}^{N} \log \left(r_{i j}\right)+\sum_{i=1}^{M} \lambda_{i}^{b}\left(\sum_{j=1}^{N} S_{i j}-G_{i}\right)+\sum_{i=1}^{M} \lambda_{i}^{r}\left(\sum_{i=1}^{N}\left(r_{i j}-H_{i}\right)\right.$

Here, $\lambda_{i}^{b}$ and $\lambda_{i}^{r}$ are Lagrangian variables. Dual problem can be stated in terms of $\lambda^{b}$ and $\lambda^{r}$ using the following equation (21):

$D_{T}\left(\lambda_{i}^{b}, \lambda_{i}^{r}\right)=\sum_{i=1}^{M}\left(\sum_{j=1}^{N} S_{i j}-G_{i}\right) \lambda_{i}^{b}+\sum_{i=1}^{M}\left(\sum_{j=1}^{N} r_{i j}-H_{i}\right) \lambda_{i}^{r}-\sum_{i=1}^{M} \sum_{j=1}^{N} \log \left(r_{i j}\right)$

where $\lambda_{i}^{b}>0$ and $\lambda_{i}^{r}>0$.

The property of duality states that the upper bound of a utility can be given as:

$\max _{S_{i j}} P\left(S_{i j}, \lambda_{i}^{b}, \lambda_{i}^{r}\right) \leq \min _{\lambda_{i}^{b} \lambda_{i}^{r}} D_{T}\left(\lambda_{i}^{b}, \lambda_{i}^{r}\right)$

Now as $\lambda_{i}^{b}$ and $\lambda_{i}^{r}$ are firmly having a value more than zero satisfying slater condition and durable duality stands true in which case the highest value of the former situation equals to the lowest value. The best possible solution $S_{i j}$ can be attained by solving best solution for $\lambda_{i}^{b}$ and $\lambda_{i}^{r}$. Now we adopt gradient method to solve the problem of duality which has two Lagrangian mutable which in turn get changed in the reverse direction of gradient $\Delta d(\lambda)$. The process is iterative in manner, with $\mathrm{Nth}$ iteration being:

a. In case of cellular user and $V_{R}$ which receive driving signals transmitted by every $B_{S}$ and $V_{T}$ each signal contains $\lambda_{i}^{b}$ and $\lambda_{i}^{r}$ where best allocation of bandwidth can be computed using derivative of $S_{i j}$ with ' $n$ ' number of iterations.

$$
\left.\begin{array}{l}
\frac{\partial I}{\partial S_{i j}(k)}=-\frac{1}{S_{i j}(k)}+\lambda_{i}^{b}(k)+\lambda_{i}^{r}(k) \log \left(1+U_{i j}\right)=0 \\
=>S_{i j}(k)=\frac{1}{\lambda_{i}^{b}(k)+\lambda_{i}^{r}(k) \log \left(1+U_{i j}\right)}
\end{array}\right\}
$$

With ' $n$ ' number of iterations all cellular users have to select the best servicing $B_{S}$ and each $V_{R}$ selects the best $B_{S}$ which satisfies the condition:

$$
j^{*}(k)=\max _{j} \frac{\log \left(1+U_{i j}\right)}{\lambda_{i}^{b}(k)+\lambda_{i}^{r}(k) \log \left(1+U_{i j}\right)}, \forall j \in \widehat{B_{S}}
$$

Where $\widehat{B_{S}}$ is set of cellular $B_{S}$

$$
j^{*}(k)=\max _{j} \frac{\log \left(1+U_{i j}\right)}{\lambda_{i}^{b}(k)+\lambda_{i}^{r}(k) \log \left(1+U_{i j}\right)}, \forall j
$$

Bandwidth allocation in concerned access area is given by:

$$
B_{i j}(k)=\frac{1}{\lambda_{i}^{b}(k)+\lambda_{i}^{r}(k) \log \left(1+U_{i j}\right)}
$$

b. Now when $B_{S}$ receives bandwidth requirement from all the cellular users as well as $V_{R}$ then $\lambda_{i}^{b}$ and $\lambda_{i}^{r}$ are refreshed and information is reverted to cellular user and $V_{R}$. 
International Journal of Mathematical, Engineering and Management Sciences

Vol. 4, No. 1, 27-40, 2019

https://dx.doi.org/10.33889/IJMEMS.2019.4.1-003

$$
\begin{aligned}
& \lambda_{i}^{b}(k+1)=\lambda_{i}^{b}(k)-\delta \frac{\partial D_{T}}{\partial \lambda_{i}^{b}(k)}=\lambda_{i}^{b}(k)-\delta\left(\sum_{j \epsilon \psi(i)} S_{i j}(k)-G_{i}\right) \\
& \lambda_{i}^{r}(k+1)=\lambda_{i}^{r}(k)-\delta \frac{\partial D_{T}}{\partial \lambda_{i}^{r}(k)}=\lambda_{i}^{r}(k)-\delta\left(\sum_{j \epsilon \psi(i)} r_{i j}(k)-H_{i}\right)
\end{aligned}
$$

Where $\psi(i)$ is set of cellular users and $V_{R}$ who choose $B_{S} . \delta>0$ is step size and is adjusted in order to achieve speedy merging of the whole process. The algorithm moves towards merging a certain number of iterations using the above equations (27) and (28). $\lambda_{i}^{b}$ and $\lambda_{i}^{r}$ could be understood as shadow concept pricing. If bandwidth increases beyond a value of threshold the associated price will also go up in order for users to link with it. Else price will decrease which in turn would attract more number of users. At each iteration the complexity of dissemination is $\mathrm{K}(\mathrm{MxN})$ and value of information exchanged is $\mathrm{K}(\mathrm{M}+\mathrm{N})$ here $\mathrm{K}$ is total number of cycles. Now provided $\mathrm{K}$ remains relatively small the algorithm performs exceedingly well in comparison to brute force in selected scenarios. When the process iteration ends the reduction in power is computed which has corresponding indicator as $\alpha_{i}$ whose value is 0 or 1 . As defined in (Sehrish et al., 2013) condition that assures merger of the dual problem is given as a derivative of $D_{T}(\lambda)$ :

$$
\begin{gathered}
\frac{\partial D_{T}}{\partial \lambda_{i}^{b}(k)}=\sum_{j \epsilon \psi(i)} S_{i j}(k)-G_{i} \\
\frac{\partial D_{T}}{\partial \lambda_{i}^{r}(k)}=\sum_{j \epsilon \psi(i)} r_{i j}(k)-H_{i}
\end{gathered}
$$

In case of optimization problem $\sum j \epsilon \varphi(i) S_{i j}$ and $\sum j \epsilon \varphi(i) r_{i j}$ are bounded in range $\left[0, G_{i}\right]$ and $\left[0, H_{i}\right]$ which means derivative of $D_{T}(\lambda)$ will also be bounded as:

$s t u_{k}\left\{\left\|\partial D_{T}(\lambda)\right\| \leq H\right.$

Where $\mathrm{H}$ is scalar in nature and satisfies condition proposition as given in (Sehrish et al., 2013). This means that assurance can be given for decomposition algorithm, which tends to merge in order to give a partial effective solution.
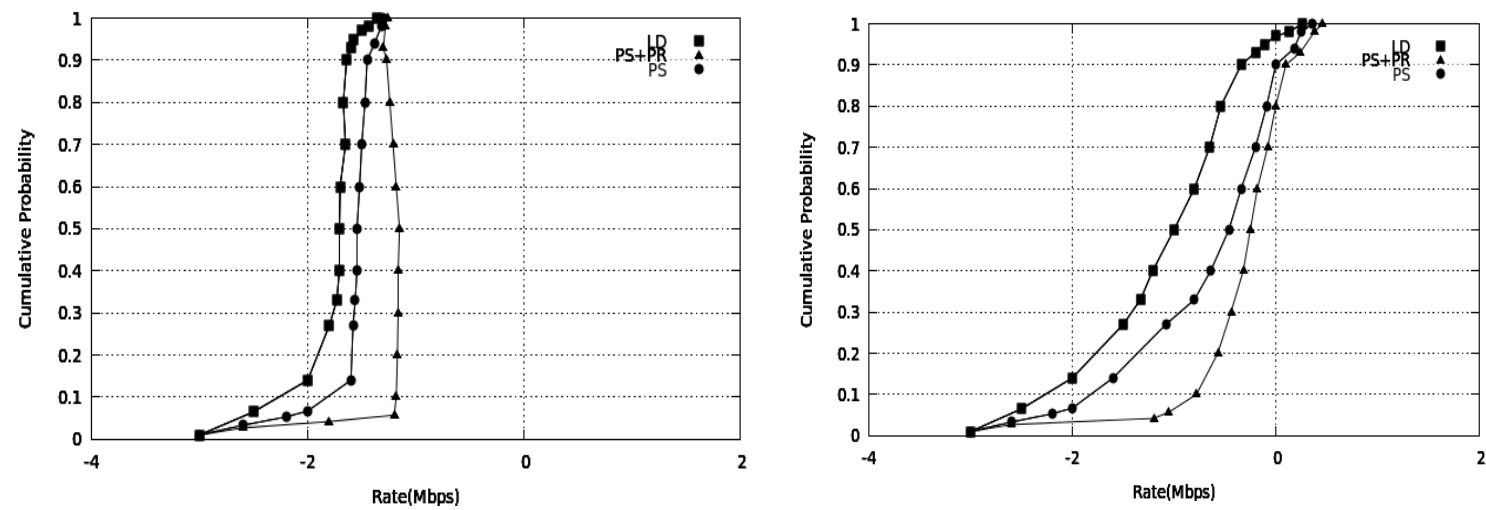

Figure 2. Throughput gain using different schemes with $\eta=1 / 4$ and $\eta=1 / 2$ 
International Journal of Mathematical, Engineering and Management Sciences

Vol. 4, No. 1, 27-40, 2019

https://dx.doi.org/10.33889/IJMEMS.2019.4.1-003

\section{Simulation Analysis}

Simulations have been conducted in NS3 and SUMO in order to demonstrate the working of the algorithm proposed. Now assuming a typical macro-cell which has a frequency equivalent to 2.1 Ghz on which it works. The region of simulation is limited to 1.24 X 1.24 (miles). Distance between two sites is $500 \mathrm{mtrs}$ and $70 B_{S}$ are deployed within the region.
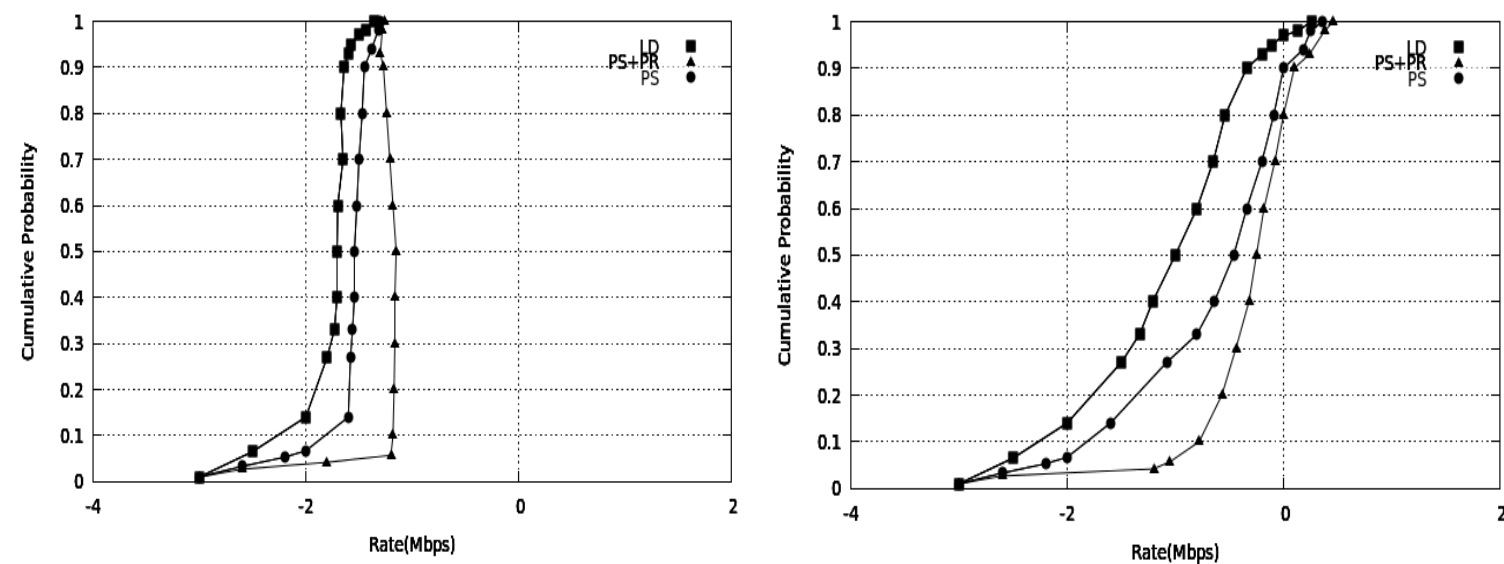

Figure 3. Throughput distribution with different schemes using different densities $\lambda=\mathbf{0 . 0 1}$ and $\lambda=\mathbf{0 . 0 2}$ respectively

The models for channels are provided by METIS as introduced in (Song and Tao, 2017) with different types of propagation. Two scenarios are explained one for cellular links and the other for vehicular links and are referred to as \#3 and \#4 respectively. Other parameters used in the simulation are: transmission power which has a value of $B_{S}=45 \mathrm{dBm}$ and $V_{T}=20 \mathrm{dBm}$. The height of antenna is $12 \mathrm{~m}$ for $B_{S}$ and $2.5 \mathrm{~m}$ for $V_{T}$. The provided bandwidth and capacity for every $V_{T}$ is $50 \mathrm{Mbps}$. The thermal noise value is at $-104 \mathrm{dBm}$ with the delay in a transmission having boundary value of $100 \mathrm{~ms}$ with a transmission range of a given $V_{T}$ being around $20 \mathrm{~m} .1300$ cellular users are distributed including $V_{R}$, vehicles are moving on roads both sides with density $\lambda$ vehicles/m and remaining are cellular users. It is considered in simulations that all cellular users are haphazardly placed and all the vehicles are haphazardly distributed on the road and vehicles move mutually having their own speed, which varies with cycles. Cycles simply show the minimal value for asset regulation for radio as an association to a given user and handoff, management of energy and so on. For each road a selected size of vehicles is taken into consideration for $V_{T}$ measured by ratio $\eta$. The vehicle density $\lambda$, ratio $\eta$ and number of roads $\left(N_{R}\right)$ are used as variables.

Table 1. Throughput Comparison between different schemes under different $\eta$

\begin{tabular}{|c|c|c|c|}
\hline Scenario & $\begin{array}{c}\text { Location Dependent } \\
\text { (LD) }\end{array}$ & $\begin{array}{c}\text { Peerless Selection } \\
\text { (PS) }\end{array}$ & $\begin{array}{c}\text { Peerless Selection with Power Reduction } \\
\text { (PS+PR) }\end{array}$ \\
\hline$\eta=1 / 4$ & 610.6 & 917 & 1291.5 \\
\hline$\eta=1 / 2$ & 628.5 & 935.2 & 1689.3 \\
\hline
\end{tabular}


International Journal of Mathematical, Engineering and Management Sciences

Vol. 4, No. 1, 27-40, 2019

https://dx.doi.org/10.33889/IJMEMS.2019.4.1-003
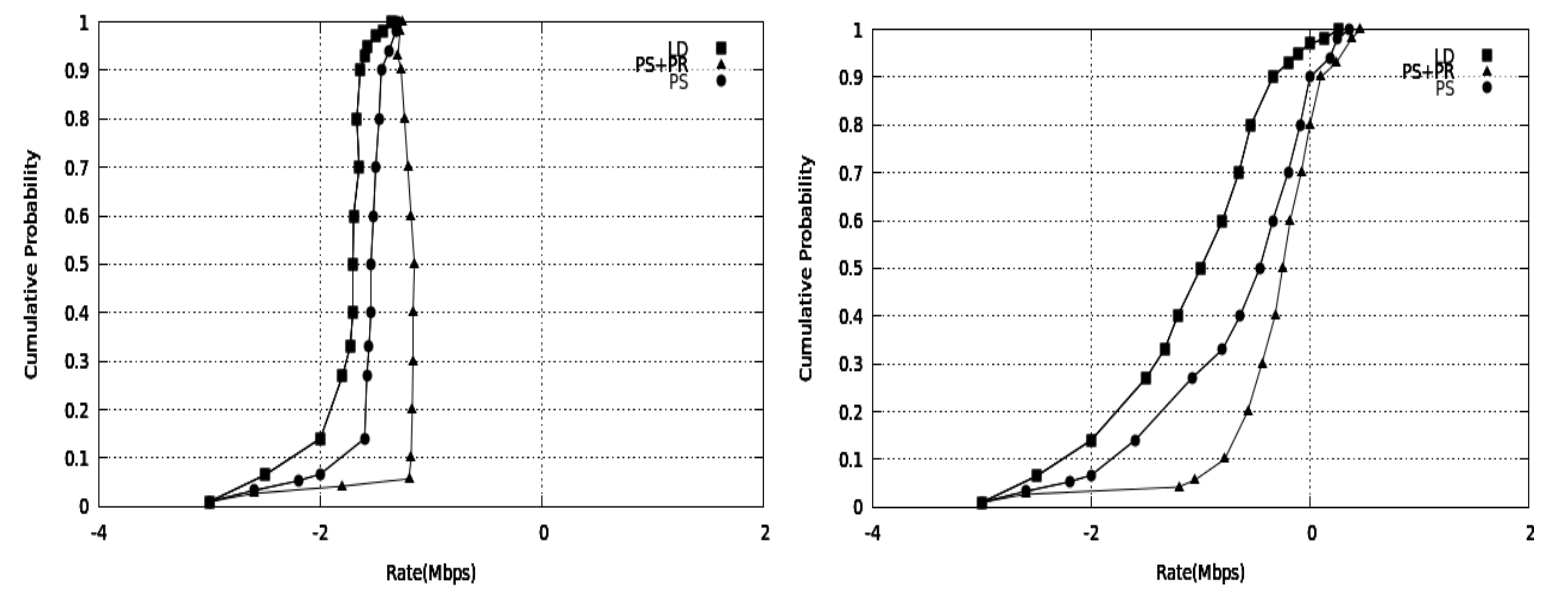

Figure 4. Throughput dissemination among schemes having varied road scenario $\boldsymbol{N}_{R}=\mathbf{3 0}$ and $\boldsymbol{N}_{R}=\mathbf{6 0}$ respectively

Throughput performance is compared under varied scenarios namely location dependent asset distribution (George and Venugopal, 2012), peerless selection without reduction in power and peerless selection with reduction in power which are denoted as LD, PS and PS+PR respectively. In case of location dependent scheme manual trials having varied division ratio are determined. By changing parametric values, we can assess the influence of corresponding parametric values such as $\eta, \lambda$ and $N_{R}$ on throughput. Shows distribution of throughput between schemas having varied ratio between value of $V_{T}$ and vehicles in totality. $N_{R}$ value is stationary and set at 30 and $\lambda$ equals to 0.02 vehicles $/ \mathrm{m}$. At the end of sixteen iterations merger is seen in Figure 2 with substantial throughput gain considering only $5 \%$ of cell edge and total throughput of $\eta=0.25$. Gain in throughput becomes more prominent when $V_{T}$ count is doubled as shown in Figure 2. Table 1 depicts collective throughput of different schemas. The PS shows $23 \%$ gain in throughput with $\eta=0.25$. When PS is combined with PR and all idle $B_{S}$ and $V_{T}$ are turned off $41 \%$ gain is achieved in comparison to without PR. The gain in throughput is even higher with $\eta=0.5$ going up to $121 \%$. Large gain is seen due to cellular users which select the best possible mode for approach and attaining maximum throughput without compromising on asset distribution equity, turning off idle $B_{S}$ and $V_{T}$ that hinder the throughput contributions towards improvement and lead to avoiding interference with enhanced throughput. However, LD can help reduce interference improves on SINR and in turn lower spectrum efficiency utilization and ultimately limits throughput gains. As shown in Figure 3 throughput distributions in the above mentioned schemes having different density $\lambda$ is compared to $N_{R}$ equals to 30 and $\eta=0.5$. High vehicle density $\lambda$ means more number of $V_{T}$ ultimately resulting in more complex interference scenario. Now as $\lambda$ will increase delay distance between any pair of vehicles will decrease. We observe substantial gains in the scenario of 5\% cellular edge and total throughput. Table 2 shows a total gain of $81 \%$ in throughput in case of the PS+PR scheme in comparison to when $\lambda=0.01$ vehicles $/ \mathrm{m}$. 
International Journal of Mathematical, Engineering and Management Sciences

Vol. 4, No. 1, 27-40, 2019

https://dx.doi.org/10.33889/IJMEMS.2019.4.1-003

Table 2. Throughput Comparison between different schemes under different $\boldsymbol{\lambda}$

\begin{tabular}{|c|c|c|c|}
\hline Scenario & $\begin{array}{c}\text { Location Dependent } \\
\text { (LD) }\end{array}$ & $\begin{array}{c}\text { Peerless Selection } \\
\text { (PS) }\end{array}$ & $\begin{array}{c}\text { Peerless Selection with Power Reduction } \\
\text { (PS+PR) }\end{array}$ \\
\hline$\lambda=0.01$ & 466 & 725.8 & 1145.6 \\
\hline$\lambda=0.02$ & 583.6 & 869.6 & 1647.7 \\
\hline
\end{tabular}

The gain percentage goes up-to $117 \%$ with $\lambda=0.0203 \frac{\text { vehicles }}{m}$ this results in the recommended schema being beneficial in throughput gain, also in scenarios of high vehicle density. Figure 4 depicts throughput dissemination amongst schemes having varied road numbers $N_{R}$. $\lambda$ and $\eta$ Set to 0.01 vehicles $/ \mathrm{m}$ and 0.5 . Road complexity is identical to interference schema as total number of $V_{T}$ increase with number of road. The figure clearly depicts the attained value of gain in throughput in case of 30 road and 60 road scenario. Table 3 shows throughput increases to $112 \%$ which depicts that the schema considered is suitable in varied road cases. Throughput performance is verified in a dynamic surrounding. As all vehicles move on the highway it leads to formation of an aggressive network structure therefore it becomes crucial to assess the behaviour. Peerless selection approach switch occurs at the beginning. At the end of operation of algorithm all $B_{S}$ and $V_{T}$ decide upon whether to continue or not in active state. While the cycle continues position of cellular user may change and vehicles may move along the highway with a predefined speed. Assuming all are disseminated haphazardly in the area and every vehicle tends to move towards a new variable position. The position is generally updated and creates a change in the network. It is assumed that $N_{R}$ equals to 30 with values of $\lambda$ equals to $0.02 \frac{\text { vehicles }}{m}$ and $\eta$ equals to 0.25 . As seen in Table 4 throughput is highest with every cycle competition which results to the fact that the scheme proposed is stable even when network is dynamic.

Table 3 : Throughput comparison between different schemes under different $\boldsymbol{N}_{\boldsymbol{r}}$

\begin{tabular}{|c|c|c|c|}
\hline Scenario & $\begin{array}{c}\text { Location Dependent } \\
\text { (LD) }\end{array}$ & $\begin{array}{c}\text { Peerless Selection } \\
\text { (PS) }\end{array}$ & $\begin{array}{c}\text { Peerless Selection with Power Reduction } \\
\text { (PS+PR) }\end{array}$ \\
\hline$N_{R}=30$ & 455.1 & 707.2 & 1118.2 \\
\hline$N_{R}=60$ & 626.1 & 911.6 & 1712.7 \\
\hline
\end{tabular}

Table 4. Throughput comparison between different schemes during five cycles (Mbps)

\begin{tabular}{|c|c|c|c|}
\hline Cycle & $\begin{array}{c}\text { Location Dependent } \\
(\text { LD) }\end{array}$ & $\begin{array}{c}\text { Peerless Selection } \\
(\text { PS) }\end{array}$ & $\begin{array}{c}\text { Peerless Selection with Power Reduction } \\
\text { (PS+PR) }\end{array}$ \\
\hline 1 & 757.9 & 1077.8 & 1596.4 \\
\hline 2 & 644.2 & 956.6 & 1474.7 \\
\hline 3 & 595.7 & 869.7 & 1311.5 \\
\hline 4 & 715.8 & 1047.6 & 1489.5 \\
\hline 5 & 740.3 & 1082.9 & 1466.4 \\
\hline
\end{tabular}


International Journal of Mathematical, Engineering and Management Sciences

Vol. 4, No. 1, 27-40, 2019

https://dx.doi.org/10.33889/IJMEMS.2019.4.1-003

\section{Conclusion}

This paper studies the peerless selection approach and asset distribution problem in which each $V_{R}$ is capable to take the decision of selecting the most appropriate approach among cellular network and VANET. The cellular users have their inherent best serving $B_{S}$. A two-phase algorithm is proposed which provides a near to perfect solution keeping the basis of convex optimization with dual decomposition adopted in order to provide user combined maximum utilization problem a possible solution by making the idle $B_{S}$ and $V_{T}$ to be switched off. This verifies that the scheme proposed achieves a substantial gain in throughput in comparison to the existing scheme with varied parameters applied.

\section{Conflict of Interest}

The authors confirm that there is no conflict of interest to declare for this publication.

\section{Acknowledgement}

The authors would like to express their sincere thanks to the referee and there valuable suggestions for the improvement of this paper.

\section{References}

Al-Kubati, G., Al-Dubai, A., Mackenzie, L., \& Pezaros, D. P. (2015, June). Stable infrastructure-based routing for intelligent transportation systems. In Communications (ICC), 2015 IEEE International Conference on (pp. 3394-3399). IEEE.

Al-Kubati, G., Al-Dubai, A., Mackenzie, L., \& Pezaros, D. P. (2014, April). Efficient road topology based broadcast protocol for VANETs. In Wireless Communications and Networking Conference (WCNC), 2014 IEEE (pp. 2710-2715). IEEE.

Alshaer, H. (2015, February). Securing vehicular ad-hoc networks connectivity with roadside units support. In GCC Conference and Exhibition (GCCCE), 2015 IEEE 8th (pp. 1-6). IEEE.

Arora, A., Rakesh, N., \& Mishra, K. K. (2018). Analysis of safety applications in VANET for LTE based network. In Networking Communication and Data Knowledge Engineering (pp. 141-154). Springer, Singapore.

Bai, Y., Xie, D., Wang, S., \& Zhong, M. (2015, October). Multi-path transmission protocol in VANET. In Connected Vehicles and Expo (ICCVE), 2015 International Conference on (pp. 204-209). IEEE.

Balasubramanian, A., Levine, B. N., \& Venkataramani, A. (2008, September). Enhancing interactive web applications in hybrid networks. In Proceedings of the 14th ACM International Conference on Mobile Computing and Networking (pp. 70-80). ACM.

Bazzi, A., \& Zanella, A. (2016). Position based routing in crowd sensing vehicular networks. Ad Hoc Networks, 36, 409-424.

Bessani, A., Correia, M., Quaresma, B., André, F., \& Sousa, P. (2013). DepSky: dependable and secure storage in a cloud-of-clouds. ACM Transactions on Storage, 9(4), 12.

Bitam, S., \& Mellouk, A. (2014). Routing for vehicular ad hoc networks. In Bio-Inspired Routing Protocols for Vehicular Ad Hoc Networks. 29-50, John Wiley \& Sons.

Di Felice, M., Ghandour, A. J., Artail, H., \& Bononi, L. (2012, July). On the impact of multi-channel technology on safety-message delivery in IEEE $802.11 \mathrm{p} / 1609.4$ vehicular networks. In Computer Communications and Networks (ICCCN), 2012 21st International Conference on (pp. 1-8). IEEE. 
International Journal of Mathematical, Engineering and Management Sciences

Vol. 4, No. 1, 27-40, 2019

https://dx.doi.org/10.33889/IJMEMS.2019.4.1-003

Elazab, M., Noureldin, A., \& Hassanein, H. S. (2017). Integrated cooperative localization for vehicular networks with partial GPS access in urban canyons. Vehicular Communications, 9, 242-253.

George, K., \& Venugopal, V. (2012, May). Design and performance measurement of a high-performance computing cluster. In Instrumentation and Measurement Technology Conference (I2MTC), 2012 IEEE International (pp. 2531-2536). IEEE.

Han, B., Hui, P., Kumar, V. S., Marathe, M. V., Pei, G., \& Srinivasan, A. (2010, September). Cellular traffic offloading through opportunistic communications: a case study. In Proceedings of the 5th ACM Workshop on Challenged Networks (pp. 31-38). ACM.

Jemaa, I. B., Shagdar, O., Martinez, F. J., Garrido, P., \& Nashashibi, F. (2015, January). Extended mobility management and routing protocols for internet-to-VANET multicasting. In Consumer Communications and Networking Conference (CCNC), 2015 12th Annual IEEE (pp. 904-909). IEEE.

Khaitiyakun, N., Sanguankotchakorn, T., \& Tunpan, A. (2014, February). Data dissemination on MANET using content delivery network (CDN) technique. In Information Networking (ICOIN), 2014 International Conference on (pp. 502-506). IEEE.

Malandrino, F., Casetti, C., Chiasserini, C. F., \& Fiore, M. (2012, June). Offloading cellular networks through ITS content download. In Sensor, Mesh and Ad Hoc Communications and Networks (SECON), 2012 9th Annual IEEE Communications Society Conference on (pp. 263-271). IEEE.

Mershad, K., \& Artail, H. (2013). A framework for secure and efficient data acquisition in vehicular ad hoc networks. IEEE Transactions on Vehicular Technology, 62(2), 536-551.

Mershad, K., Artail, H., \& Gerla, M. (2012a). We can deliver messages to far vehicles. IEEE Transactions on Intelligent Transportation Systems, 13(3), 1099-1115.

Mershad, K., Artail, H., \& Gerla, M. (2012b). ROAMER: Roadside Units as message routers in VANETs. Ad Hoc Networks, 10(3), 479-496.

Mostafa, A., Vegni, A. M., Singoria, R., Oliveira, T., Little, T. D., \& Agrawal, D. P. (2011, August). A V2X-based approach for reduction of delay propagation in vehicular Ad-Hoc networks. In ITS Telecommunications (ITST), 2011 11th International Conference on (pp. 756-761). IEEE.

Nam, J., Min, S., \& Kim, S. (2015). Extended wireless mesh network for VANET with geographical routing protocol. 11th International Conference on Wireless Communications, Networking and Mobile Computing (WiCOM 2015).

Puspitorini, O., Siswandari, N., Mahmudah, H., \& Wijayanti, A. (2017). Measurement of interconnecting network for roadside unit placement on cellular network to support intelligent transportation system. Asian Journal of Applied Sciences, 5(2), 247-258.

Ramakrishnan, L., Canon, R. S., Muriki, K., Sakrejda, I., \& Wright, N. J. (2012). Evaluating interconnect and virtualization performance for high performance computing. ACM SIGMETRICS Performance Evaluation Review, 40(2), 55-60.

Sehrish, S., Mackey, G., Shang, P., Wang, J., \& Bent, J. (2013). Supporting HPC analytics applications with access patterns using data restructuring and data-centric scheduling techniques in MapReduce. IEEE Transactions on Parallel and Distributed Systems, 24(1), 158-169.

Song, W., \& Tao, X. (2017, September). Analysis of a Location-Aware Probabilistic Strategy for Opportunistic Vehicle-to-Vehicle Relay. In Vehicular Technology Conference (VTC-Fall), 2017 IEEE 86 th (pp. 1-6). IEEE. 